\title{
Photonic liquid crystal fiber intermodal interferometer
}

\section{Interferómetro intramodal de fibra de cristal fotónico con cristal líquido}

\author{
David Poudereux ${ }^{(1, *)}$, Pedro Corredera(2,S) Eva Otón(1), José M. Otón(1), Xabier Quintana(1) \\ 1. CEMDATIC, ETSI Telecomunicación, Universidad Politécnica de Madrid, Av. Complutense, 30, 28040 Madrid, Spain. \\ 2. CSIC, Instituto de Óptica, C/ Serrano, 144, 28006, Madrid, Spain. \\ (*) Email: dpoudere@gmail.com S: miembro de SEDOPTICA / SEDOPTICA member \\ Received / Recibido: 20/09/2013. Revised / Revisado: 07/10/2013. Accepted / Aceptado: 08/10/2013. \\ DOI: http://dx.doi.org/10.7149/OPA.46.4.321
}

\begin{abstract}
:
In this work, we present a novel interferometer based on liquid crystal and photonic crystal fiber technology. The objective of this project is the development of a tunable (switchable) modal (MachZehnder) interferometer for optical communications or sensing. This interferometer has been manufactured splicing a short portion (between 15 and $30 \mathrm{~mm}$ ) of photonic crystal fiber with two single mode fiber pigtails. The study shows a high sensitivity of the interferometer to the polarization of the launching light..
\end{abstract}

Key words: Mach-Zehnder Interferometer, Liquid Crystal, Photonic Crystal Fiber, Polarization Maintaining Fiber, Tunable Interferometer.

\section{RESUMEN:}

En este trabajo se presenta un nuevo interferómetro basado en fibras de cristal fotónico con cristal líquido. El objetivo de este estudio es el desarrollo de un interferómetro intermodal (tipo MachZehnder) sintonizable, útil para comunicaciones ópticas y para sensores. Este interferómetro ha sido fabricado soldando una pequeña porción de fibra de cristal fotónico a dos pigtail de fibra estándar monomodo. El estudio muestra que el interferómetro tiene una sensibilidad muy alta a la poloarización de la luz acoplada.

Palabras clave: Interferómetro Mach-Zehnder, Cristal Líquido, Fibra de Cristal Fotónico, Fibra Mantenedora de Polarización, Interferómetro Sintonizable.

\section{REFERENCES AND LINKS / REFERENCIAS Y ENLACES}

[1]. J. C. Knight, T. A. Birks, P. St. Russel, D. M. Atkin, "All-silica single-mode optical fiber with photonic crystal cladding", Opt. Lett. 21, 1547-1549 (1996). DOI

[2]. J. C. Knight, "Photonic crystal fibres", Nature 424, 847-851 (2003). DOI

[3]. S. Ertman, A.-H. Rodríguez, M. M. Tefelskja, M. S. Chychłowski, D. Pysz, R. Buczyński, E. NowinowskiKruszelnicki, R. Dąbrowski, T. R. Woliński, "Index guiding photonic liquid crystal fibers for practical applications", J. Lightwave Tehnol. 30, 1208-1214 (2012). DOI

[4]. T. P. Hansen, J. Broeng, E. B. Libori, E. Knudsen, A. Bjarklev, J. R. Jensen, H. Simonsen, "Highly birefringent index-guiding photonic crystal fibers", IEEE Phot. Technol. Lett. 13, 588-590 (2001). DOI

[5]. K. Suzuki, H. Kubota, S. Kawanishi, M. Tanaka, M. Fujita, “Optical properties of a low-loss polarizationmaintaining photonic crystal fiber", Opt. Express 9, 676-680 (2001). DOI

[6]. O. Frazão, J. Viegas, P. Caldas, J. L. Santos, F. M. Araújo, L. A. Ferreira, F. Farahi, "All-fiber MachZehnder curvature sensor based on multimode interference combined with a long-period grating", Opt. Lett. 32, 3074-3076 (2007). DOI

[7]. H. Y. Choi, M. J. Kim, B. H. Lee, “All-fiber Mach-Zehnder type interferometers formed in photonic crystal fiber", Opt. Express 15, 5711-5720 (2007). DOI 
[8]. L.-T. Hsiao, C.-P. Yu, "Mach-Zehnder fiber interferometers based on liquid-filled photonic crystal fibers", Proc. 21 st Ann. IEEE Wireless Opt. Comm. Conf. WOCC'12, pp. 146-148 (2012).

[9]. J. Du, Y. Dai, G. K. P. Lei, W. Tong, C. Shu, "Photonic crystal fiber based Mach-Zehnder interferometer for DPSK signal demodulation”, Opt. Express 18, 7917-7922 (2010). DOI

[10]. B. Dong, J. Hao, Z. Xu, "Temperature insensitive curvature measurement with a core-offset polarization maintaining photonic crystal fiber based interferometer", Opt. Fiber Technol. 17,233235 (2011). DOI

[11]. M. Deng, C.-P. Tang, T. Zhu, Y.-J. Rao, "Highly sensitive bend sensor based on Mach-Zehnder interferometer using photonic crystal fiber", Opt. Commun. 284, 2849-2853 (2011). DOI

[12]. O. Frazao, C. Jesus, J. M. Baptista, J. L. Santos, P. Roy, “Fiber-optic interferometric torsion sensor based on a two-LP-mode operation in birefringent fiber", IEEE Phot. Tech. Lett. 21, 1277-1279 (2009). DOI

[13]. J. Villatoro, V. Finazzi, G. Badenes, V. Pruneri, "Highly sensitive sensors based on photonic crystal fiber modal interferometers", J. Sensors 2009, 747803 (2009). DOI

\section{Introduction}

Photonic Crystal Fibers (PCFs) are microstructured fibers with periodically distributed air holes in the cladding region. These air holes can be infiltrated with liquid or gases. PCFs may feature solid core guiding by modified Total Internal Reflection (mTIR) or hollow core, whose guiding mechanism is by Photonic BandGap (PBG) transmitting only certain wavelengths values [1-3]. Some sorts of PCFs have several cores or holes of different diameters in order to improve specific properties as endlessly modes or polarization maintaining [4,5]. These properties may be further boost by infiltrating in the holes a birefringent medium such a liquid crystal.

In this work, a Polarization Maintaining PCF (PM-1550) of NKT Photonics has been employed. This fiber shows two opposite wider holes around the non-circular core that creates strong birefringence (Fig.1).

Taking advantage of the differences in the hole diameters it has been possible to collapse and filling selectively either the wider or the narrower holes with liquid crystals (LC). This opens the possibility of creating fiber-based tunable interferometers.

In this work is presented two different interferometers, one surrounded by LC and other partially filled in his wider cavities with LC. In both has been studied the sensitivity of the output power due to the orientation of the polarized launched light and the external applied electric field.

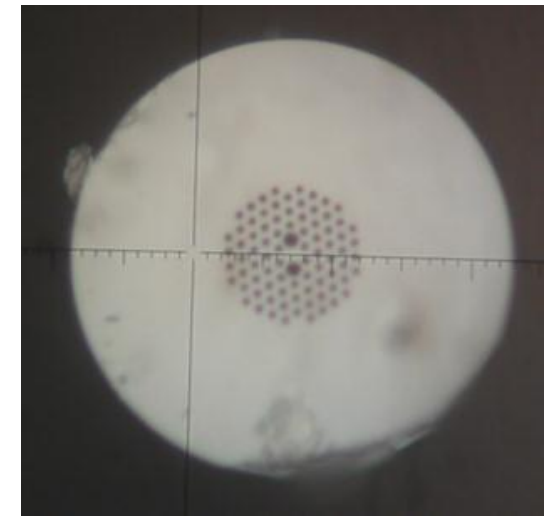

Fig. 1. PM-1550 manufactured by NKT Photonics.

\section{Mach-Zehnder interferometer}

By introducing collapsed regions or offset splicing at the splicing points of a PCF and two single-mode fibers (SMFs), one can create a Mach-Zehnder Interferometer (MZI) [6,7]. Both the core mode and cladding mode can be simultaneously induced at one splicing point, propagating along different optical paths. The modes are recombined at the second splicing point, and interference patterns can arise at the output (Fig. 2). Previous studies have demonstrated that liquid-filled PCF MZIs can be successfully utilized in sensing applications of bending and surrounding refractive index and signal modulation [8-11].

Actually the liquid crystal need not be infiltrated into the microchannels to produce tunable effects. PCFs are very sensitive to their environment. Therefore a switchable PCF-MZI device may be developed merely surrounding the fiber MZI externally with the liquid crystal. 


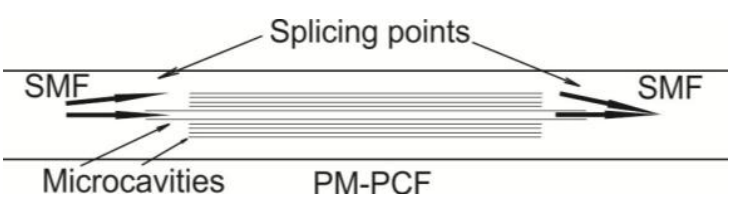

Fig. 2: Scheme of a MZI-PCF.

In this way, standard LC cells have been prepared using ITO-coated glasses and SMFs as spacers. The LC molecular director can be reoriented by an electric field applied between the two glass plates. Initially the LC is aligned homogeneously. After applying a sufficiently high electric field, the alignment switches to homeotropic.

The selected PCF works on a two-LP-mode operation. When LP light is launched at $45^{\circ}$ relatively to the eigenaxes of the high birefringent $\mathrm{PCF}$, both polarization states of the two spatial modes $\mathrm{LP}_{01(x y)}$ and $\mathrm{LP}_{11(x y)}$ are simultaneously excited, generating a multibeam interference [12].

\section{Fabrication process}

\subsection{Fusion splicing}

The splicing points of the interferometer are critical because the cladding mode is induced or recombined within these regions. The fusion process has been realized using an Ericcson Fusion Splicer FSU-900.

Two separate splicing processes had to be developed. The fusion of an empty PCF with a SMF to induce cladding modes requires to fully collapse the intermediate region. If the PCF is totally or selectively filled with a LC, the fusion process must be made with extreme care to avoid LC decomposition. In this case the current intensity must be much lower; otherwise bubbles are formed inside the fusion region.

Alignment is also important in both cases, because it determines the power balance between the two paths of the interferometer (Fig. 3).

In the case of MZIs with externally surrounded LC, both splicing points were completely collapsed.

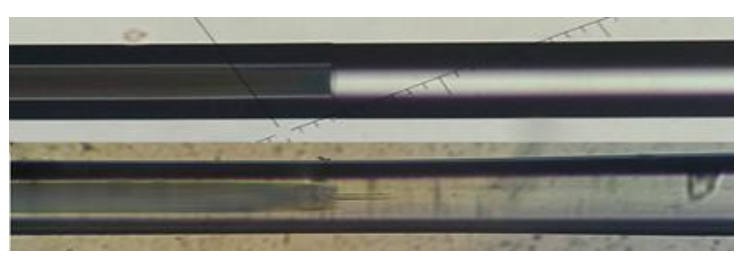

Fig. 3. PCF selectively filled with LC fusioned superficially with SMF (top) and PCF-SMF totally collapsed fusion (bottom).

\subsection{Liquid Crystal encapsulation}

The interferometer has been encapsulated between two ITO-coated glasses using SMFs as spacers. In this way, an electric field across the fibers may be applied by applying voltage to the ITO electrodes. Both glasses have been preconditioned to anchor the LC molecules parallel to the surface in absence of an electric field. When a field is applied to the cell, the LC molecules will reorient to become parallel to the field (i.e., perpendicular to the glasses). Upon reorientation the LC intends to reach a minimum energy state that depends on the field strength and the anchoring force that try to keep the molecules parallel to the substrates. This reorientation can be therefore modulated, making it possible to modify the effective refractive index 'seen' by the evanescent field of the propagating light waves.

Due to selective filling the $\mathrm{PCF}$, an initial partial collapsing of the smaller cavities whit the fusion splicer has been made, remaining only the two wider cavities. After the filling process in vacuum, the filled PCF is cut and spliced with the second single mode pigtail.

The alignment of LC molecules in infiltrated PCF is more complex. Alignment preconditioning of the inner microchannel surfaces inside the fiber proved to be unfeasible. Alignment is achieved by taking ad-vantage of the LC viscous anisotropy, by which a LC flowing in a capillary spontaneously orients along the flow direction. Therefore molecules become homogeneously oriented, parallel to the walls of the cavity. When an electric field is applied, molecules tend to align orthogonally to the walls, parallel to the electric field lines (Fig. 4).

Homeotropic alignment (i.e., perpendicular to the walls) has been tried as well, with the 


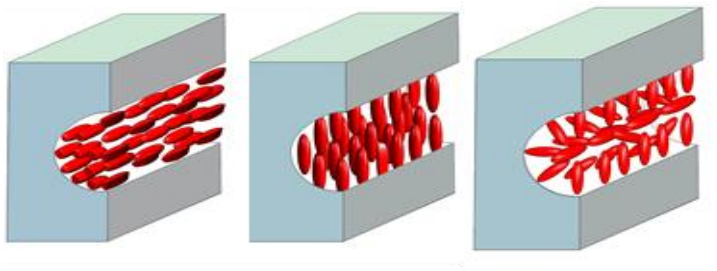

Fig. 4. Homogeneous alignment of LC molecules (left), perpendicular alignment with applied electric field (center) and radial third-dimension escape (right).

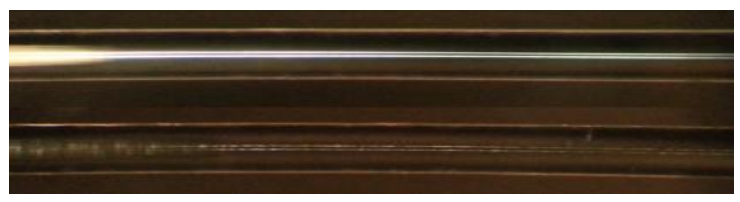

Fig. 5. PM-PCF with his wider cavities filled with MLC-1300 between polarizers at $45^{\circ}$ to one (top) and parallel (bottom).

added difficulties mentioned above. Although homeotropic alignment could be attractive for a number of applications, the outcome is quite involved in practice. Indeed, homeotropic conditioning of the microchannel walls does not lead to a symmetric radial structure but rather to an escaped radial structure, with a third dimension escape, whereby the energetically disfavored molecular interactions at the center of the capillary are avoided. Even in the ideal case the third dimension escape is rarely a homogenous structure since there are two degenerate opposite escape directions, giving rise to domain formation and scattering.

It's difficult to observe the alignment of the LC molecules inside a specific cavity of the fiber through the microscope because of the scattering of the whole cladding.

The used method has been to focus on the collapsed region of the spliced point where it is possible to see the two wider cavities that are not collapsed yet. Using crossed polarizers it's possible to observe the LC alignment by changing the orientation of the fiber from parallel to $45^{\circ}$ respect to the polarizer (Fig. 5).

\section{Characterization}

\subsection{MZI surrounded by LC}

Two tunable lasers in the $1450-1550 \mathrm{~nm}$ and 1510-1650 nm bands have been used for measurements and characterization. Lasers show a wavelength dependent elliptical quasilinear polarization. The polarization of the impinging light had to be controlled and monitored in order to maintain it constant throughout the whole measurement.

Several MZIs with different lengths were fabricated in order to observe the dependence between the MZI length and the period of the pattern. It turns out that the period is inversely proportional to the length as shown in Fig. 6.

The period or fringe spacing $\Delta \lambda$ of these interferometers is

$$
\Delta \lambda=\frac{\lambda^{2}}{\Delta n_{e f f} L},
$$

being $\Delta n_{e f f}$ the effective refractive index difference between the modes participating in the interference [13].

In the measurement of the MZI surrounded by LC a circular polarization was set due to his wavelength independence inducing a multibeam interference. The two sets of interference patterns, corresponding to two orthogonal polarizations, are superimposed, resulting in an amplitude-modulated wave (Fig. 7).

It is easy to see how the amplitude increases in the higher-amplitude zone when voltage is applied and how the phase changes in the lower amplitude zone. In fact this change in phase only is showed in the lower-amplitudes zones while no change is visible in the higher.

Applying the Fast Fourier Transform it is possible to obtain the relationship between the frequency of the spectrum fringes and their amplitudes. The two set of peaks showed in Fig. 8 represent the two-LP-mode interferometer between $\mathrm{LP}_{01(x y)}$ and $\mathrm{LP}_{11(x y)}$.

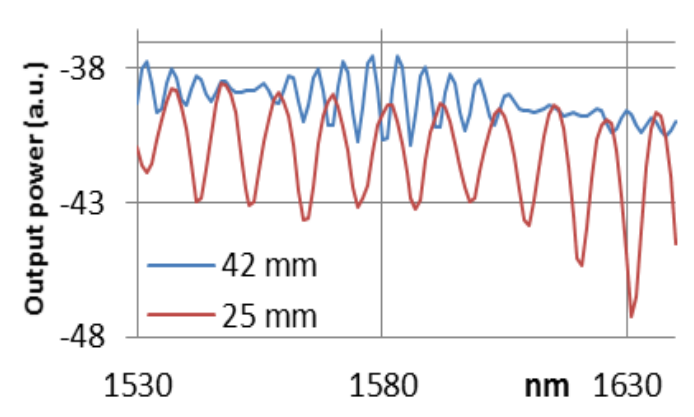

Fig. 6. Dependence of fringe spacing with MZI length. 


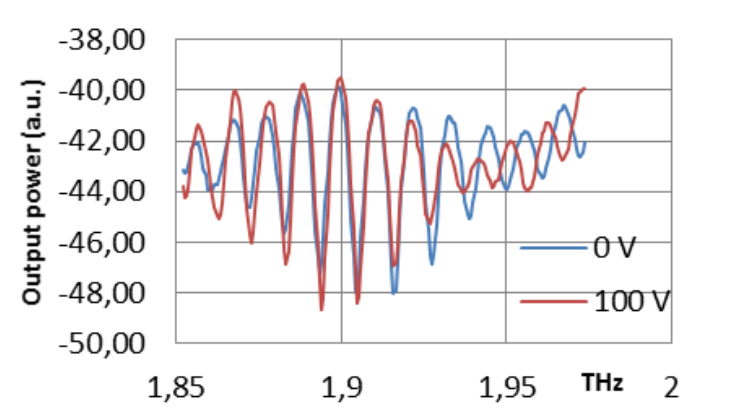

Fig. 7. Interference pattern of a Mach-Zehnder interferometer of $42 \mathrm{~mm}$ surrounded by MLC-13000 LC.

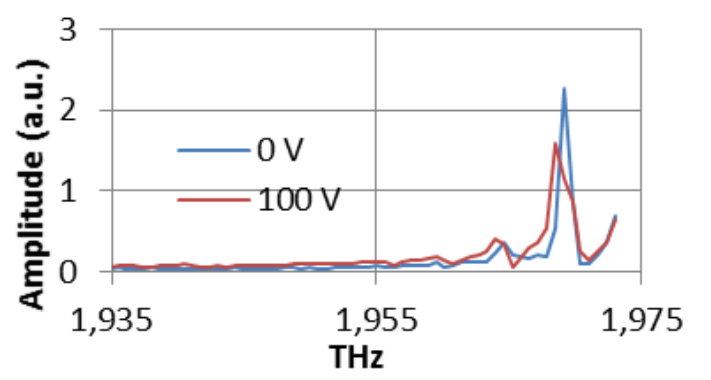

Fig. 8. FFT of the interferometer with and without applied electric field.

The amplitude changes with the orientation of the input polarization. Each peak represents the interferometry of modes of the same $\mathrm{x}$ or $\mathrm{y}$ axis. It is possible to see that at least one higher mode has been excited (the second peak in Fig. 8).

\subsection{MZI with LC in his wider microcavities}

In the interferometer filled selectively with LC, the variation of the pattern when an electric field is applied is much more abrupt, although the interference pattern is not as clear as the previous one. The setup is much more sensible to the polarization. A band-gap is detected for certain voltage values as the axis along the cavities filled with LC is guided by PBG rather than MTIR like the orthogonal axis.

A band-gap is observed in the absence of electric field (@ 1600nm, see Fig. 10). The bandgap performs a hypochromic shift (about $25 \mathrm{~nm}$ ) when an $80 \mathrm{~V}$ voltage is applied; while disappears when voltage is rised up to $120 \mathrm{~V}$.

Measurements were taken on the same setup using circular polarized light (Fig. 9) and linear polarized light afterwards (Fig. 10).

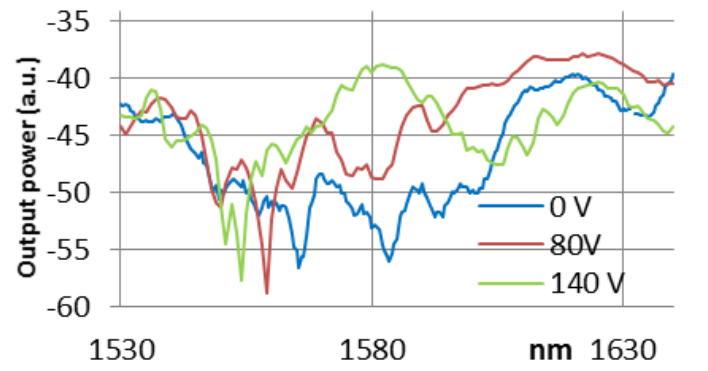

Fig. 9. MZI of $16 \mathrm{~mm}$ filled with LC selectively at various applied electric fields launched with circular polarized light.

\section{Conclusions}

In this study the possibility of manufacturing a tunable photonic liquid crystal Mach-Zehnder Interferometer has been demonstrated. The MZI is sensible to polarization and voltage controllable.

MZIs for different applications based on the same design can be prepared by changing several properties as the MZI length, the type of PCF or Micro Structured Fiber and the type of Liquid Crystal. If third-dimension escape of homeotropic samples could be effectively controlled, this orientation associated to negative electric anisotropy LCs would be interesting for inducing polarization-insensitive axial symmetric LC reorientations.

\section{Acknowledgements}

This work has been supported by the Spanish Ministerio de Ciencia e Innovación, project TEC2008-00147 and Madrid Regional Program FACTOTEM S2009/ESP1781. 